\title{
UN HALLAZGO INUSUAL: UN NÚCLEO DE ÓPALO Y CALCEDONIA EN LA CUENCA MEDIA DEL RÍO GALLEGOS (SANTA CRUZ, ARGENTINA)
}

\author{
FLAVIA CARBALLO M. ${ }^{\text {a }}$ JUAN BAUTISTA BELARDI ${ }^{\mathrm{b}} \&$ PEDRO TIBERI ${ }^{\mathrm{a}}$
}

\section{RESUMEN}

En el sector sur de un amplio cañadón denominado Mack Aike (conocido localmente como Chorrillo Grande) -margen norte de la cuenca media del río Gallegos-, se registró un voluminoso núcleo (231 mm x 210 $\mathrm{mm} \times 147 \mathrm{~mm}$ ) con extracciones multidireccionales producto de la talla de un rodado tamaño bloque de ópalo y calcedonia. En el marco de la tecnología regional del Campo Volcánico Pali Aike (CVPA) el hallazgo de este núcleo es inusual. Se describe la génesis geológica y las características petrográficas macroscópicas del bloque y aquellas tecnológicas del núcleo. El bloque es de origen glaciario y habría estado disponible localmente. Pese a lo atractivo de las materias primas que conforman el núcleo, sus fracturas y discontinuidades lo tornaron de regular y mala calidad para la talla. Esto explica que el $50 \%$ de las terminaciones sean quebradas y en charnela y que el $38,23 \%$ de las lascas obtenidas hayan sido corticales. Además, la amplia distribución y disponibilidad local de materias primas aptas para la talla habría hecho que no se produjera una explotación más intensa del núcleo. Su aprovechamiento fue oportunista y se lo abandonó.

PALABRAS CLAVE: cuenca media del río Gallegos, Campo volcánico Pali Aike, ópalo, calcedonia.

\section{AN UNUSUAL FINDING: AN OPAL AND CHALCEDONY CORE IN THE GALLEGOS RIVER MIDDLE BASIN (SANTA CRUZ, ARGENTINA)}

\begin{abstract}
On the Southern sector of a wide canyon called Mack Aike (locally known as Chorrillo Grande) located on the northern margin of the middle Gallegos River, a bulky core (231 mm x $210 \mathrm{~mm}$ x 147 $\mathrm{mm}$ ) with multidirectional scars from knapping an opal and chalcedony block-sized nodule was registered. Within the framework of the regional technology of the Pali Aike Volcanic Field, the finding of this core is unusual. The geological genesis and the macroscopic petrographic characteristics of the block and the technological characteristics of the core are described. The block has a glacial origin and would have been

a Universidad Nacional de la Patagonia Austral, Unidad Académica Río Gallegos (ICASUR). $\measuredangle$ flaviacarballomarina@gmail.com; pedro.tiberi@gmail.com

b Universidad Nacional de la Patagonia Austral, Unidad Académica Río Gallegos (ICASUR), Consejo Nacional de Investigaciones Científicas y Técnicas (CONICET). juanbautistabelardi@gmail.com
\end{abstract}


locally available. Despite the attractiveness of the raw materials that make up the core, its fractures and discontinuities turned it of regular and poor knapping quality. This explains that $50 \%$ of the terminations are broken and forming hinges and that $38.23 \%$ of the flakes obtained have been cortical. In addition, the wide distribution and local availability of suitable lithic raw materials contributed to a less intensive exploitation of the core. Its use was opportunistic and it was abandoned.

KEY WORDS: Gallegos River middle basin, Pali Aike Volcanic Field, opal, chalcedony.

\section{INTRODUCCIÓN}

Los muestreos de materias primas aptas para la talla llevados a cabo en el sector argentino del Campo Volcánico Pali Aike (CVPA) dan cuenta de una gran variabilidad en la distribución y disponibilidad de rocas (Nami, 1984; Charlin, 2009; Borrazzo et al. 2017, entre otros). Como parte de un estudio de impacto ambiental sobre el registro arqueológico se relevó un amplio cañadón denominado Mack Aike (conocido localmente como Chorrillo Grande) ( 21 km), ubicado en el sector medio de la margen norte del río Gallegos (Fig. 1). Sobre el lado sur y oeste y en el borde y el piso del mallín del cañadón se reconoció una distribución artefactual relativamente continua con variaciones en su densidad que permiten proponer un uso recurrente del cañadón por parte de las poblaciones cazadoras recolectoras. Las ocupaciones alcanzaron hasta momentos históricos, tal el caso del sitio Chorrillo Grande 1 -en estudio- donde se registraron raspadores y cuentas de vidrio, artefactos de latón y restos de caballo (Equus caballus), seguramente en función de la oferta de reparo, agua y concentración de guanacos (Lama guanicoe).

Hacia el sur del cañadón Mack Aike, sobre un camino interno de la estancia Alquinta, se registró un voluminoso núcleo $(231 \mathrm{~mm}$ x $210 \mathrm{~mm}$ x $147 \mathrm{~mm}$ ) con extracciones multidireccionales producto de la talla de un rodado tamaño bloque de ópalo y calcedonia (Figs. 1 y 2). Se encontraba semienterrado unos $6-8 \mathrm{~cm}$ en un sector de alta densidad artefactual. En los alrededores no se halló ninguna de las lascas obtenidas a partir del núcleo. No obstante, hay desechos de talla y raederas confeccionadas en otras rocas disponibles localmente.

En el CVPA la disponibilidad de calcedonia, que puede proceder tanto de fuentes primarias

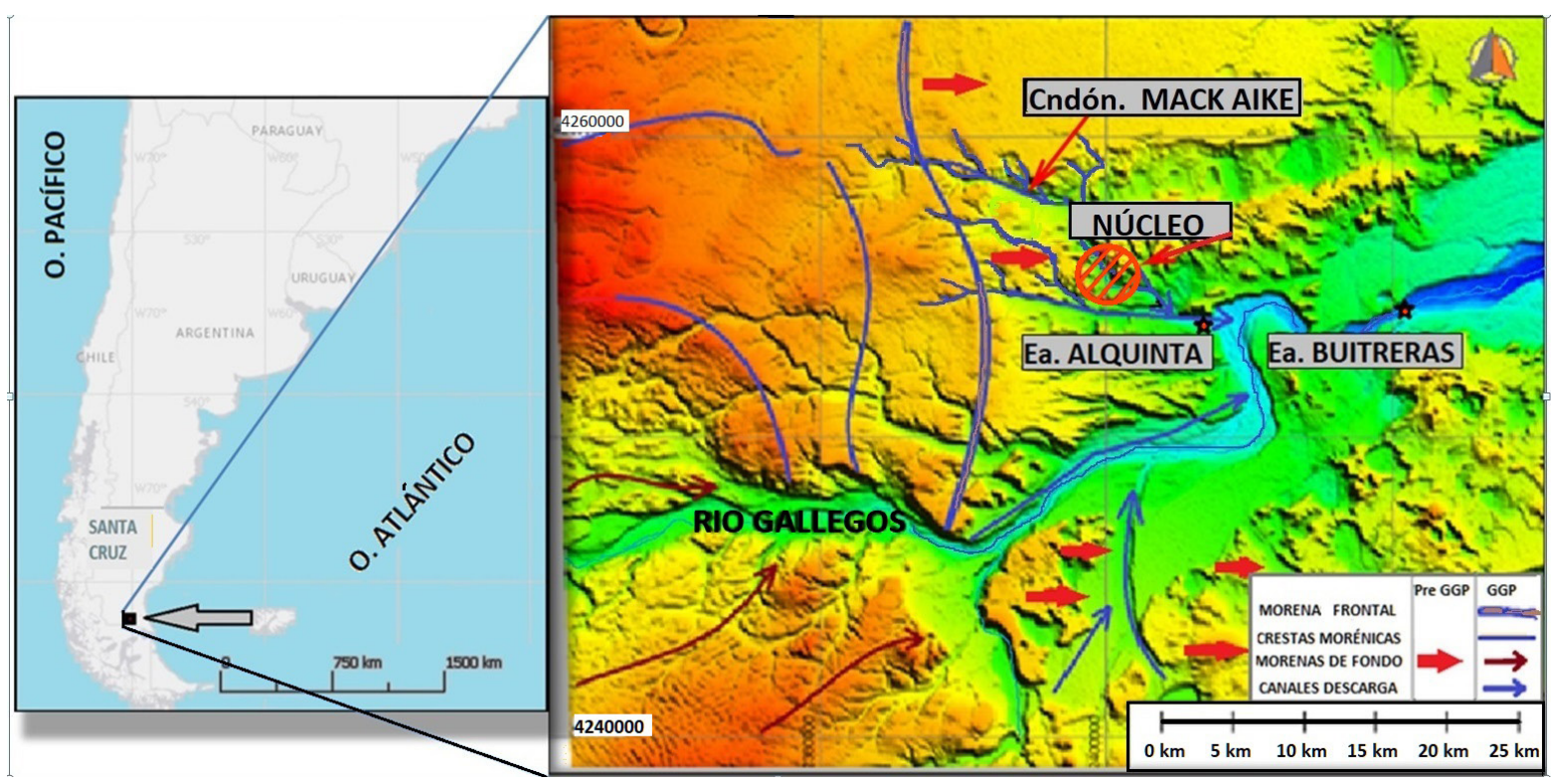

Fig. 1. Modelo de elevación digital mostrando los diferentes cordones morénicos Pre GGP y GGP con sus planicies de outwash (modificado de Ercolano et al. 2015). 


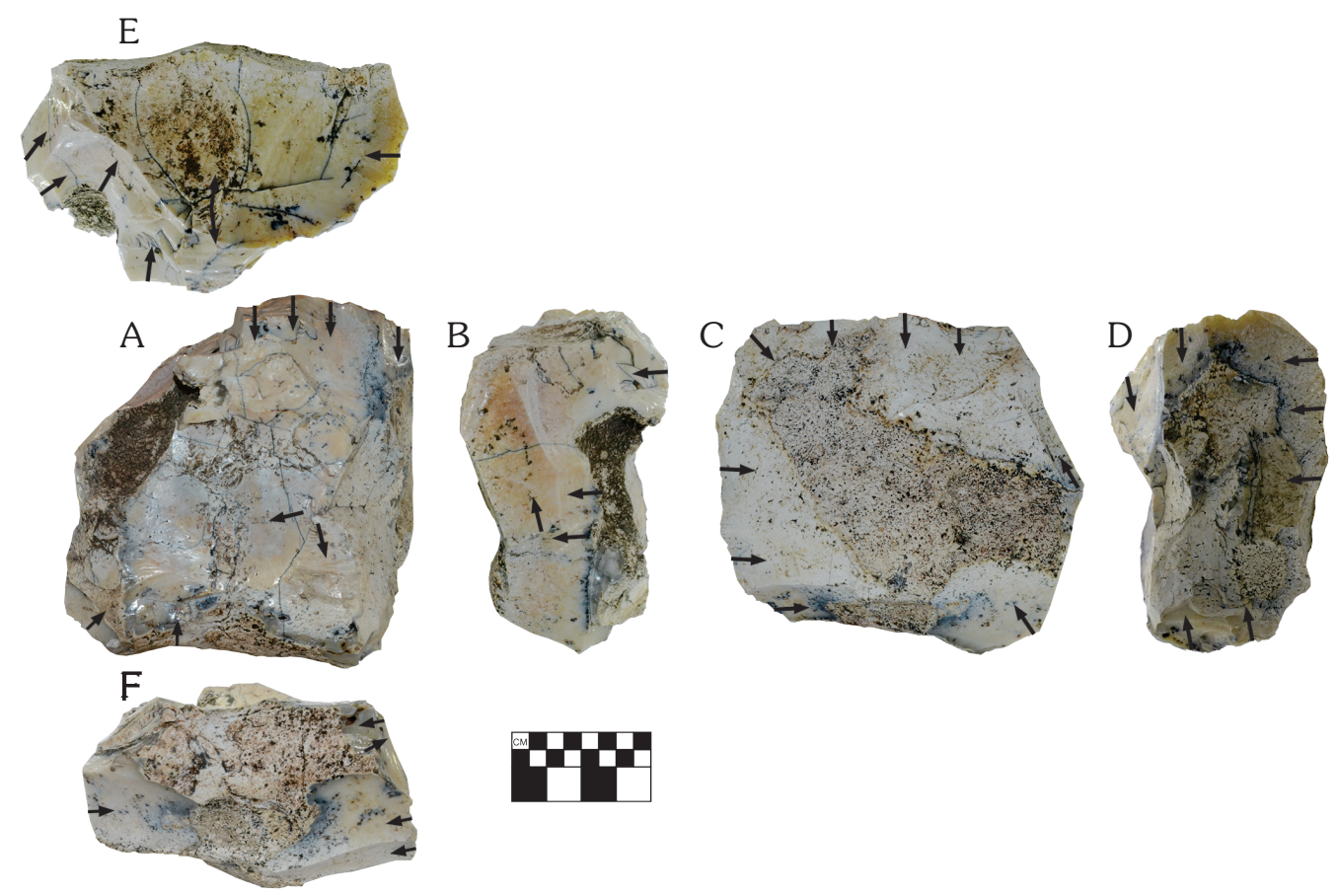

Fig. 2. Núcleo. Caras (A, B, C y D) giradas en el sentido de las agujas del reloj, E: vista superior, $\mathrm{F}$ : vista inferior. Las flechas indican la orientación de las distintas extracciones.

como secundarias, es $\leq 13 \%$ y ocupa el tercer lugar en la manufactura de los artefactos, luego de las Rocas Oscuras de Grano Fino (RGFO) y la dacita; ha sido detectada en un solo muestreo en el sector sur del CVPA (Charlin, 2009). En el sitio La Carlota cueva 1 es la roca predominante debido a la presencia de una potencial fuente secundaria -en forma de plaquetas de entre 4 y $5 \mathrm{~cm}$ - que se encuentran sobre la cara $\mathrm{SO}$ de la colada donde se ubica la cueva (Campan et al. 2007). La frecuencia de artefactos de calcedonia sugirió que se encontraría disponible en el sector central del CVPA (Charlin, 2009). Una situación semejante sucede con la disponibilidad de fuentes de ópalo ( $\leq 3 \%$ ), aunque se desconoce su procedencia. Además, los artefactos manufacturados en dichas materias primas son los que presentan los menores tamaños (con una media de 13,78 y 14,82 mm de largo, respectivamente) (Charlin, 2009).

El hecho de que el núcleo haya sido confeccionado sobre un bloque de ópalo y calcedonia hace del mismo un hallazgo inusual. Se describe el origen geológico y las características petrográficas macroscópicas del bloque y aquellas tecnológicas del núcleo. Luego, se evalúan las implicaciones del mismo dentro del contexto del uso de materias primas líticas dentro del CVPA.

\section{ORIGEN Y CARACTERÍSTICAS PETROGRÁFICAS MACROSCÓPICAS DEL BLOQUE}

En el interfluvio Gallegos-Coyle -área del hallazgo- se reconoce una serie de depósitos de origen glaciar atribuidos a la glaciación pedemontana de mayor extensión hacia la Patagonia extrandina, denominada Gran Glaciación Patagónica (GGP) (Mercer, 1976) o Glaciación Bella Vista (Meglioli, 1992), fechada entre 1,15 y 1,05 Ma (Ton-That et al. 1999). El paisaje está constituido por una gran variedad de geoformas depositacionales vinculadas con la acción directa del hielo proveniente del SO y de las corrientes glacifluviales que se desplazaron sobre un sustrato friable de rocas sedimentarias miocenas pertenecientes a la Formación Santa Cruz (Ercolano et al. 2015). Además, indican la coexistencia de glaciaciones Pre-GGP, con depósitos de diseño lobular que se proyectan en dirección $\mathrm{NE}$; con un patrón de depositación tipo concéntrico o anidado. Dichos depósitos están constituidos por una sucesión 
de cordones y arcos morénicos relativamente continuos y bien definidos, identificándose rocas propias del ambiente cordillerano chileno (Ercolano et al. 2015). En planta exhiben forma festoneada y son intercalados por planicies glacifluviales estrechas (Fig. 1). Asimismo, se reconocen morenas de fondo de crestas redondeadas con numerosos bloques erráticos y una sucesión de crestas morénicas estrechamente apiladas y muy disectadas por acción hídrica de magnitud. Se las han vinculado con períodos de deglaciación y dieron como resultado planicies glacifluviales o de outwash. Los materiales finos de las morenas y de las planicies glacifluviales fueron arrastrados por el agua de fusión que se encauzó en los numerosos cañadones que desembocan en el río Gallegos y dejaron en superficie solo aquellos rodados o bloques resistentes y de mayor tamaño provenientes de cordillera. Tal es el caso del bloque que fue base del núcleo bajo estudio (Fig. 2).

El 95\% del bloque es ópalo blanco grisáceo a pardo claro, con motas oscuras debido a la presencia de óxidos de hierro y en menor medida manganeso. Posee una textura grumosa, con sombras de brechamiento grueso dando lugar a una matriz heterogénea, oquerosa, con brillo vítreo a seroso, untuoso. Muestra una serie de fracturas y discontinuidades reunidas en tres grupos principales según su orientación (Fig. 3). El primero de ellos está formado por grietas rectilíneas paralelas entre sí y separadas por varios centímetros. El siguiente intercepta al anterior en un ángulo cercano a los $90^{\circ}$ y el tercero lo hace de forma irregular a curvada. Todas ellas muestran un relleno de sílice gris oscura de menos de $1 \mathrm{~mm}$, de grosor y boxworks de pirita. Aún se observa una carcasa o costra exterior que copió la forma del hueco donde se alojó la solución silícea que dio lugar a la constitución del bloque (Fig. 3). El $5 \%$ restante lo conforma una sílice calcedónica, de baja temperatura, blanca grisácea a traslúcida, con leve bandeamiento que muestra -en parte- huecos que generan discontinuidades. Es una calcedonia de grano muy fino ubicada sobre los bordes externos del bloque y revestida de una capa milimétrica de ópalo blanco homogéneo, masivo. En ocasiones muestra sombras de fragmentos de sillice más clara, con tonalidades pardo claro, dando lugar a una matriz heterogénea.

La materia prima posee dureza $\leq 7$ en la escala de Mohs que le imprime la propia sílice y que la hace difícil de rayar o marcar pero muy frágil

Tabla 1. Características del bloque (modificado de Aragón \& Franco, 1997).

\begin{tabular}{ccccc}
\hline $\begin{array}{c}\text { Textura de la } \\
\text { matriz }\end{array}$ & $\begin{array}{c}\text { Cristales, } \\
\text { vesículas, } \\
\text { oquedades }\end{array}$ & Tipo de roca/\% & Observaciones & Calidad para la talla \\
\hline $\begin{array}{c}\text { Gruesa } 0,1 \mathrm{a} \\
5 \mathrm{~mm}\end{array}$ & $2 \%$ & Ópalo/95\% & $\begin{array}{c}\text { Óxidos de hierro, } \\
\text { boxworks de pirita }\end{array}$ & Regular a mala \\
$\begin{array}{c}\text { Fina } 0,01 \mathrm{a} \\
0,1 \mathrm{~mm}\end{array}$ & $0,5 \%$ de $3 \mathrm{~mm}$ & Calcedonia/5\% & $\begin{array}{c}\text { Parches de posibles } \\
\text { sulfuros }\end{array}$ & Regular a mala \\
\hline
\end{tabular}
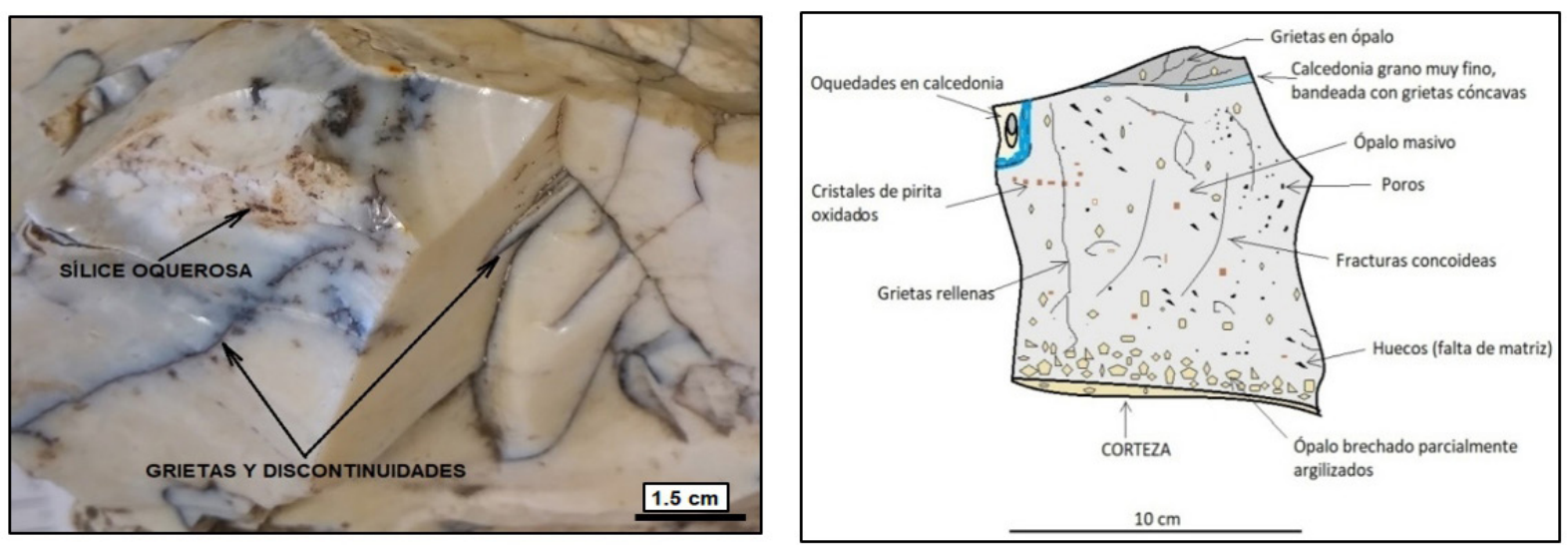

Fig. 3. Fotografía y esquema de un sector del bloque que muestran las discontinuidades. 
Tabla 2. Características de las plataformas de lascado y las lascas.

Referencias: no dif.: no diferenciada.

Los largos entre paréntesis indican que esa dimensión está fracturada.

\begin{tabular}{|c|c|c|c|c|c|c|c|}
\hline \multicolumn{2}{|c|}{$\begin{array}{l}\text { No Plataforma de lascado/ } \\
\text { Estado/ Forma }\end{array}$} & \multirow{2}{*}{$\begin{array}{c}\text { Lasca } \\
1\end{array}$} & \multirow{2}{*}{$\begin{array}{c}\begin{array}{c}\text { Tipo de } \\
\text { lasca }\end{array} \\
\text { interna }\end{array}$} & \multirow{2}{*}{$\begin{array}{c}\text { Terminación } \\
\text { charnela }\end{array}$} & \multirow{2}{*}{$\begin{array}{c}\begin{array}{c}\text { Largo } \\
(\mathrm{mm})\end{array} \\
82,5\end{array}$} & \multirow{2}{*}{$\begin{array}{c}\begin{array}{c}\text { Ancho } \\
(\mathrm{mm})\end{array} \\
68\end{array}$} & \multirow{2}{*}{$\begin{array}{l}\text { Tamaño } \\
\text { grande }\end{array}$} \\
\hline \multirow{8}{*}{ 1/Activa } & lisa & & & & & & \\
\hline & lisa & 3 & interna & quebrada & 25,5 & 20 & pequeño \\
\hline & lisa & 4 & interna & aguda & 74 & 68 & mediano/grande \\
\hline & no dif. & 5 & cortical & aguda & $(31,5)$ & 22 & pequeño \\
\hline & no dif. & 6 & cortical & aguda & $(31)$ & 56 & pequeño \\
\hline & lisa & 7 & cortical & aguda & 49 & 47 & mediano/pequeño \\
\hline & lisa & 8 & interna & aguda & 19,5 & 10,5 & pequeño \\
\hline & lisa & 9 & interna & aguda & 29 & 15,5 & mediano/pequeño \\
\hline 2/ Activa & lisa & 1 & interna & charnela & 70 & 105,5 & mediano/grande \\
\hline \multirow{3}{*}{ 3/ Activa } & no dif. & 1 & interna & quebrada & (35) & 64,5 & pequeño \\
\hline & filiforme & 2 & cortical & aguda & 30 & 68 & pequeño \\
\hline & filiforme & 3 & cortical & aguda & 68,5 & 44,5 & mediano/grande \\
\hline \multirow{4}{*}{ 4/ Activa } & natural & 1 & interna & charnela & 40 & 46,5 & pequeño \\
\hline & filiforme & 2 & interna & quebrada & 39 & 50,5 & pequeño \\
\hline & filiforme & 3 & interna & quebrada & 22 & 22 & pequeño \\
\hline & lisa & 4 & interna & quebrada & 23,5 & 50,5 & pequeño \\
\hline \multirow{6}{*}{ 5/ Activa } & natural & 1 & cortical & quebrada & 32 & 51,5 & pequeño \\
\hline & natural & 2 & cortical & quebrada & 79 & 71,5 & mediano/grande \\
\hline & natural & 3 & cortical & quebrada & 30 & 35,5 & pequeño \\
\hline & natural & 4 & cortical & quebrada & 90,5 & 40,5 & grande \\
\hline & natural & 5 & cortical & aguda & 85,5 & 50 & grande \\
\hline & natural & 6 & cortical & aguda & 17,5 & 25,5 & muy pequeño \\
\hline \multirow{4}{*}{ 6/Agotada } & facetada & 1 & interna & quebrada & 52 & 46 & mediano/pequeño \\
\hline & natural & 2 & interna & aguda & 27,5 & 30,5 & pequeño \\
\hline & natural & 3 & interna & charnela & 66 & 86 & mediano/grande \\
\hline & natural & 4 & interna & aguda & 68 & 86 & mediano/grande \\
\hline \multirow{2}{*}{ 7/ Activa } & puntiforme & 1 & interna & aguda & 76,5 & 55,5 & mediano/grande \\
\hline & puntiforme & 2 & interna & aguda & 44 & 51,5 & mediano/pequeño \\
\hline \multirow{2}{*}{ 8/ Agotada } & puntiforme & 1 & interna & aguda & 74 & 91 & mediano/grande \\
\hline & puntiforme & 2 & interna & aguda & 64 & 43 & mediano/grande \\
\hline \multirow{4}{*}{ Sin plataforma } & no dif. & 1 & interna & charnela & $(66,5)$ & 41,5 & mediano/grande \\
\hline & no dif. & 2 & interna & aguda & (66) & 13 & mediano/grande \\
\hline & no dif. & 3 & cortical & quebrada & $(26,5)$ & 34 & pequeño \\
\hline & no dif. & 5 & cortical & quebrada & $(75,5)$ & 82 & mediano/grande \\
\hline
\end{tabular}


ante un golpe. Esto se debe a la matriz compleja y a las discontinuidades que generan los planos de fractura irregular (Tabla 1 y Fig. 3).

\section{CARACTERÍSTICAS TECNOLÓGICAS DEL NÚCLEO}

Se evalúan las características del núcleo según criterios propuestos por Aschero (1975/83). El núcleo es de forma poliédrica, tiene un peso de 6.076 gr y un volumen de $2.600 \mathrm{~cm}^{3}$. Sólo se han extraído lascas por percusión directa y no se observan pátinas diferenciales. En sus seis caras posee un número mínimo de 34 extracciones multidireccionales generadas sobre seis plataformas activas y dos agotadas donde se han obtenido dos lascas a partir de un mismo golpe. Hay cuatro lascas que no tienen plataforma (eliminadas por extracciones previas) (Fig. 2 y Tabla 2). El 50\% de las superficies de las plataformas son preparadas. Dos de las caras del núcleo conservan un $50 \%$ de corteza (Fig. 2 C y F). Al respecto, hay 13 $(38,23 \%)$ claros negativos de lascado que indican el descortezamiento del núcleo y, por lo tanto, las extracciones fueron consideradas corticales. El porcentaje de lascas con terminaciones quebradas y en charnela alcanza el 50\% (Tablas 1, 2 y Fig. 3). Hay una variación marcada en el tamaño de las lascas que oscila entre muy pequeño $(0,1-20 \mathrm{~mm})$ y grande (80,1- $120 \mathrm{~mm}$-aunque ninguna lasca supera los $90,5 \mathrm{~mm})$. La mayor representación corresponde al rango mediano grande $(35,29 \%)$ y al pequeño (41,17\%). Los anchos también exhiben variabilidad (entre 10,5 mm y 105,5 mm) (Tabla 2).

\section{CONCLUSIÓN}

Se ha propuesto que en el CVPA la obtención de calcedonia habría sido producto de viajes de aprovisionamiento específicos dentro de una estrategia de uso planificado del espacio (Charlin, 2009). Lo mismo aplica para el ópalo. El único espacio donde la calcedonia se registra en mayor frecuencia es en el sitio La Carlota cueva 1 -al inicio de las ocupaciones (1.070 años AP)- y en virtud de la presencia de la mencionada fuente secundaria (Campan et al. 2007).

Dado que regionalmente no hay evidencia de artefactos formatizados ni de lascas de calcedonia y ópalo de grandes tamaños, el registro de un núcleo voluminoso en estas rocas constituye en sí mismo un hallazgo relevante en el CVPA por lo inusual. También para los cazadores recolectores debió ser un evento impredecible por lo que el aprovechamiento del bloque fue oportunista. $\mathrm{Su}$ volumen remanente indicaría que el bloque proviene de las proximidades del cañadón.

La ausencia de las lascas obtenidas -al menos las de mayor tamaño- apunta a su transporte. Esto, junto con las dimensiones del núcleo, podría reflejar en parte una estrategia de aprovisionamiento de lugares (Kuhn, 1995), lo que también tiene sentido si se piensa al cañadón como un lugar utilizado bajo redundancia genérica (Dewar \& McBride, 1992). Esto último se sostiene sobre la relativa continuidad de la distribución de materiales arqueológicos a lo largo de toda su extensión ( 21 $\mathrm{km})$. Ahora bien, las discontinuidades y fracturas del bloque dan como resultado que la calidad para la talla del núcleo sea regular a mala, situación que se relacionaría con que la mitad de las lascas tengan terminaciones quebradas y en charnela, su variabilidad de tamaños y la ausencia de aquellas muy grandes. Por lo tanto, su potencial de uso se restringiría a la obtención de módulos pequeños (K. Borrazzo com. pers. 2018). No obstante, el alto porcentaje de corteza en dos de las caras del núcleo sumado a que la mayoría de las plataformas de percusión están activas y, que tampoco se haya buscado reconocer su interior partiéndolo para ver si existían fragmentos utilizables, indica que no se avanzó más en su aprovechamiento y fue abandonado.

El bloque habría sido un hallazgo fortuito y como núcleo fue utilizado sin mayor planificación, rotándolo en búsqueda de plataformas de lascado aunque sin descortezarlo en su totalidad. Por último, y como se mencionara, la baja representación de la calcedonia y el ópalo en los contextos arqueológicos del CVPA indica que la impronta de la explotación del núcleo sobre la tecnología regional del CVPA debió ser mínima.

\section{AGRADECIMIENTOS}

Al geólogo Oscar Mansilla de ENAPSIPETROL Argentina y a los Sres. Ignacio de Urquiza, Joaquín Díaz y demás personal de 
estancia Alquinta por su ayuda durante el trabajo de campo. De la misma manera, agradecemos a la Lic. Patricia Campan y al Sr. Pablo Binaghi.

Karen Borrazzo, Silvana Espinosa y Luis Borrero realizaron valiosos comentarios. Además, Silvana Espinosa y Patricia Escola nos ayudaron con distintos aspectos tecnológicos.

A los evaluadores que con sus sugerencias ayudaron a mejorar el manuscrito.

\section{BIBLIOGRAFÍA}

Aragón, E., \& Franco, N. V. (1997). Características de rocas para la talla por percusión y propiedades petrográficas. Anales del Instituto de la Patagonia, Serie Ciencias Humanas, 25, 187-200.

Aschero, C. (1975 revisión 1983). Ensayo para una clasificación morfológica de artefactos líticos aplicada a estudios tipológico-comparativos. Consejo Nacional de Investigaciones Científicas y Técnicas (CONICET). Ms.

Borrazzo, K., L'Heureux, G. L., Cirigliano, N., Pallo, M.C., Ozán, I., Manzi, L., \& Charlin, J. (2017). Arqueología del interfluvio Gallegos-Chico (Santa Cruz, Argentina): nuevas prospecciones. Trabajo presentado en las Décimas Jornadas de Arqueología de la Patagonia. Libro de Resúmenes. (pp. 78). Puerto Madryn.

Campan, P., Carballo Marina, F., \& Manzi, L. (2007). Arqueología de Estancia La Carlota (Campo volcánico Pali Aike, Argentina). En F. Morello, M. Martinic, A. Prieto \& G. Bahamonde (Eds.), Levantando piedras, desenterrando huesos... y develando arcanos, (pp.
687-699). Punta Arenas: Ediciones CEQUA.

Charlin, J. (2009). Estrategias de aprovisionamiento y utilización de las materias primas líticas en el campo volcánico Pali Aike (Prov. Santa Cruz, Argentina). BAR International Series, No. 1901. Oxford: Archaeopress.

Dewar, R. E., \& McBride, K.A. (1992). Remnant Settlement Patterns. Space, Time, and Archaeological Landscapes. En J. Rossignol y L. Wandsnider (Eds.), (pp. 227-256). New York: Plenum Press.

Ercolano, B., Tiberi, P., Marderwald, G., Coronato, A., \& Corbella, H. (2015). Morfología glacial pedemontana en el interfluvio de los ríos Coyle y Gallegos, Patagonia Austral, Argentina. VI Congreso Argentino de Cuaternario y Geomorfología. Libro de Resúmenes (pp. 221-222). Ushuaia.

Kuhn, S. L. (1995). Mousterian Lithic Technology. An Ecological Perspective. Princeton University Press.

Meglioli, A. (1992). Glacial geology of Southernmost Patagonia, the Strait of Magellan and Northern Tierra del Fuego. (Tesis inédita de Doctorado). Lehigh University, Bethlehem, U.S.A.

Mercer, J. (1976). Glacial history of Southernmost South America. Quaternary Research, 6, 125-166.

Nami, H. G. (1984). Análisis tipológico de los instrumentos provenientes del sitio "El Volcán" c.4, cuenca del río Chico, (provincia de Santa Cruz). Investigaciones prehistóricas en la cuenca del río Chico (provincia de Santa Cruz). PREP. Informes de Investigación, 1, 53-82.

Ton-That, T., Singer, B., Möner, N., \& Rabassa, J. (1999). Datación de lavas basálticas por ${ }^{40} \mathrm{Ar} /{ }^{39} \mathrm{Ar}$ y geología glacial de la región del Lago Buenos Aires. Revista de la Asociación Geológica Argentina, 54, 333-352. 